\title{
CHARACTERISTIC DISTRIBUTIONS ON 4-DIMENSIONAL ALMOST COMPLEX MANIFOLDS
}

\author{
BORIS S. KRUGLIKOV \\ Department of Mathematics and Statistics \\ University of Tromsø \\ 9037 Tromsø, Norway \\ E-mail:kruglikov@math.uit.no
}

\begin{abstract}
In this paper the Nijenhuis tensor characteristic distributions on a non-integrable four-dimensional almost complex manifold is investigated for integrability, singularities and equivalence.
\end{abstract}

1. Introduction. For a non-integrable four-dimensional almost complex manifold we will canonically define a distribution $\Pi^{2}$ by the Nijenhuis tensor $N_{J}$. In Section 2 we complete the description [K1] of invariants of an almost complex structure in dimension four, using this distribution. In Sections 3-4 we describe singularities of $\Pi^{2}$. We show they are standard if our field of planes is considered as a distribution, but they become quite specific if it is considered as a differential system.

In Sections 5-6 we study moduli and hyperbolicity of the germ of a neighborhood of a pseudoholomorphic curve. Section 7 is devoted to a geometric meaning of the integrability of the Nijenhuis tensor characteristic distribution $\Pi^{2}$ and its relation to a question of V. Arnold.

In $[\mathrm{HH}]$ Hirzebruch and Hopf proved the following topological result: If a 4-dimensional manifolds admits a rank 2 distribution, it admits an almost complex structure as well. Moreover if the manifold admits two almost complex structures, defining opposite orientations, then it admits a rank 2 distribution.

We associate a rank 2 distribution to a non-integrable almost complex structure, realizing the above topological correspondence (to one side) canonically on the differential level. Note that any almost complex structure on a 4-dimensional manifold can be perturbed to be non-integrable outside a discrete set.

2000 Mathematics Subject Classification: Primary 53C15, 35A20; Secondary 58D27, 32H15.

The paper is in final form and no version of it will be published elsewhere. 
2. Local classification of almost complex structures in dimension 4. Let $(M, J \in \operatorname{Aut}(T M))$ be an almost complex manifold of dimension $4, J^{2}=\mathbf{- 1}$. Its Nijenhuis tensor is the following $(2,1)$-tensor

$$
N_{J} \in \Lambda^{2} T^{*} M \otimes T M, \quad N_{J}(\xi, \eta)=[J \xi, J \eta]-J[J \xi, \eta]-J[\xi, J \eta]-[\xi, \eta] .
$$

Integrability of $J$ is expressed via it as $N_{J}=0([\mathrm{NW}])$.

This tensor satisfies the property $N_{J}(J \xi, \eta)=N_{J}(\xi, J \eta)=-J N_{J}(\xi, \eta)$ and so can be considered as an antilinear map $N_{J}: \Lambda^{2} \mathbb{C}^{2} \rightarrow \mathbb{C}^{2}, \mathbb{C}^{2}=\left(T_{x} M^{4}, J\right)$. The image is invariant under $J$ and if $N_{J} \neq 0$ it is a complex line $\mathbb{C} \subset \mathbb{C}^{2}$.

Thus in the domain where the structure $J$ is non-integrable a canonical distribution is obtained:

Definition 1. We call $\Pi^{2}=\operatorname{Im} N_{J} \subset T M$ the Nijenhuis tensor characteristic distribution on a 4-dimensional almost complex manifold $\left(M^{4}, J\right)$.

This distribution $\Pi^{2}$ is in general situation non-integrable. Therefore it has a nontrivial derivative $\Pi^{3}=\partial \Pi^{2}$, which is defined as the differential system with $C^{\infty}(M)$ module of sections $\mathcal{P}_{3}=C^{\infty}\left(\Pi^{3}\right)$ generated by the self-commutator of the submodule $\mathcal{P}_{2}=C^{\infty}\left(\Pi^{2}\right) \subset \mathcal{D}(M): \mathcal{P}_{3}=\left[\mathcal{P}_{2}, \mathcal{P}_{2}\right] . \Pi^{3}$ is not a distribution everywhere and its singularities form a stratified submanifold $\Sigma_{1}^{2}$ of codim $=2$.

The distribution $\Pi^{3}$ on $M \backslash \Sigma_{1}^{2}$ is generically non-integrable, so that $\partial \Pi^{3}=T M$ (or $\left.\left[\mathcal{P}_{2}, \mathcal{P}_{3}\right]=\mathcal{D}(M)\right)$ outside a stratified submanifold $\Sigma_{2}^{2}$ of codim $=2$.

If $x \notin \Sigma_{1}^{2}$ then $\Pi_{x}^{2} \subset \Pi_{x}^{3}$ has a transversal measure. In fact since the $J$-antilinear isomorphism $N_{J}\left(\cdot, \xi_{3}\right): \Pi_{x}^{2} \rightarrow \Pi_{x}^{2}$ is orientation reversing, there exist vectors $\xi_{1}, \xi_{2} \in \Pi_{x}^{2}$, $\xi_{3} \in \Pi_{x}^{3} \backslash \Pi_{x}^{2}$ such that $N_{J}\left(\xi_{1}, \xi_{3}\right)=\xi_{1}, N_{J}\left(\xi_{2}, \xi_{3}\right)=-\xi_{2}$. These $\xi_{1}, \xi_{2}$ are defined up to multiplication by a constant, while $\xi_{3}\left(\bmod \Pi_{x}^{2}\right)$ is defined up to multiplication by \pm 1 . Therefore $\Pi^{3} / \Pi^{2}$ is normed. By a similar reason $T_{x} M / \Pi_{x}^{3}$ is normed outside $\Sigma_{1}^{2}$ via the vector $\xi_{4}=J \xi_{3}$.

Note that $\Pi_{x}^{3} / \Pi_{x}^{2}$ is oriented. Actually $\left[\xi_{1}, \xi_{2}\right]\left(\bmod \Pi_{x}^{2}\right)$ depends only on the values of $\xi_{1}, \xi_{2}$ at the point $x$. It is a vector $f \xi_{3}\left(\bmod \Pi_{x}^{2}\right)$ for some $f$. So if we require $\xi_{2}=J \xi_{1}$ then $\xi_{3}$ can be chosen so that $f>0$. This produces a coorientation on $\Pi_{x}^{2} \subset \Pi_{x}^{3}$ and then via $J$ a coorientation on $\Pi_{x}^{3} \subset T_{x} M$.

Moreover the requirement $f=1$ determines canonically vector field $\xi_{1}$ (still however up to \pm 1 ) and hence $\xi_{2}=J \xi_{1}$. Then we set $\xi_{3}=\left[\xi_{1}, \xi_{2}\right]$ and $\xi_{4}=J \xi_{3}$. So the pair $\left(\xi_{1}, \xi_{2}\right)$ is defined canonically up to a sign and the pair $\left(\xi_{3}, \xi_{4}\right)$ is absolutely canonical. The following statement generalizes Theorem 7 [K1]:

THEOREM 1. Let an almost complex structure $J$ be of general position. Then at a generic point $x \in M^{4}$ the canonical frame $\left(\xi_{1}, \xi_{2}, \xi_{3}, \xi_{4}\right)$ is defined. It restores uniquely the almost complex operator $J$ and the tensor $N_{J}$ by the tables:

\begin{tabular}{|c||c|}
\hline$X$ & $J X$ \\
\hline \hline$\xi_{1}$ & $\overline{\xi_{2}}$ \\
\hline$\xi_{2}$ & $-\xi_{1}$ \\
\hline$\xi_{3}$ & $\xi_{4}$ \\
\hline$\xi_{4}$ & $-\xi_{3}$ \\
\hline
\end{tabular}

\begin{tabular}{|c||c|c|c|c|}
\hline$N_{J}(\uparrow, \leftarrow)$ & $\xi_{1}$ & $\xi_{2}$ & $\xi_{3}$ & $\xi_{4}$ \\
\hline \hline$\xi_{1}$ & 0 & 0 & $\xi_{1}$ & $-\xi_{2}$ \\
\hline$\xi_{2}$ & 0 & 0 & $-\xi_{2}$ & $-\xi_{1}$ \\
\hline$\xi_{3}$ & $-\xi_{1}$ & $\xi_{2}$ & 0 & 0 \\
\hline$\xi_{4}$ & $\xi_{2}$ & $\xi_{1}$ & 0 & 0 \\
\hline
\end{tabular}


Note that reducing a geometric structure to a frame ( $\{e\}$-structure) solves completely the equivalence problem. The idea is as follows. Consider the moduli of the problem, i.e. functions $c_{j k}^{i}$ given by the formula $\left[\xi_{j}, \xi_{k}\right]=\sum c_{j k}^{i} \xi_{i}$. Denote by $\mathbb{A}=\left\{c_{j k}^{i}\right\}$ the space of all invariants and by $\Phi: M \rightarrow \mathbb{A}$ the "momentum map" $x \mapsto\left\{c_{j k}^{i}(x)\right\}$. Then two equivalent structures have the same images and the equivalence follows. See $[\mathrm{S}]$ for more details.

3. Singularities of a Nijenhuis tensor characteristic distribution. A distribution $V=V_{1}$ is called completely non-holonomic if one of its successive derivatives $V_{i}=\partial V_{i-1}$ equals the whole tangent bundle $T M$ and the minimal such $i=r$ is called the degree of non-holonomy (can vary from point to point). The growth vector of a distribution at a point $x \in M$ is the sequence of the dimensions $\left(\mathrm{rk}_{x} V_{1}, \ldots, \mathrm{rk}_{x} V_{r(x)}\right)$.

Generically a Nijenhuis tensor characteristic distribution is completely non-holonomic outside a discrete subset in $M$. In an open dense set the growth vector is $(2,3,4)$. Then it is an Engel distribution, which has the following local normal form ([E]):

$$
\Pi^{2}=\left\langle\xi_{1}=\partial_{3}, \xi_{2}=\partial_{4}-x_{3} \partial_{2}-x_{2} \partial_{1}\right\rangle ; \quad \partial_{i}:=\partial / \partial x_{i} .
$$

Locally this $\Pi^{2}$ can be realized as a Nijenhuis tensor characteristic distribution $([\mathrm{K} 2])$. In fact, consider two transversal symmetries of the distribution: $\eta_{1}=\partial_{1}, \eta_{2}=\partial_{2}-x_{4} \partial_{1}$. Define the almost complex structure by the formula

$$
J \xi_{1}=\varphi \xi_{2}, J \eta_{1}=\eta_{2} ; \quad \varphi \neq 0 .
$$

Then one easily checks that $\operatorname{Im} N_{J}=\Pi^{2}$ whenever $\left(\partial_{\eta_{1}} \varphi\right)^{2}+\left(\partial_{\eta_{2}} \varphi\right)^{2} \neq 0$.

Moreover the following statement holds:

Proposition 2. Let $\Pi$ be an analytic distribution of rank 2 in $\mathbb{R}^{4}$. Then it can be locally realized as a Nijenhuis tensor characteristic distribution.

Proof. Let $\Pi^{2}$ be generated by $\xi_{1}=\partial_{3}$ and $\xi_{2}=\partial_{4}+h_{1} \partial_{1}+h_{2} \partial_{2}$. A pair of generators can always be chosen in such a form. Consider $\xi_{2}$ as a vector field in $\mathbb{R}^{3}\left(x_{1}, x_{2}, x_{4}\right)$ depending on a parameter $x_{3}$. It has two independent symmetries $\eta_{1}, \eta_{2} \in \mathcal{D}\left(\mathbb{R}^{3}\right):\left[\eta_{i}, \xi_{2}\right]=0$. Let us differentiate these fields by the parameter: $\partial_{3} \eta_{i}=\left[\partial_{3}, \eta_{i}\right]=a_{i}^{j} \eta_{j}+b_{i} \xi_{2}$.

Define the almost complex structure by the formula

$$
J \xi_{1}=\varphi \xi_{2}, J \eta_{1}=\alpha \eta_{1}+\beta \eta_{2} ; \quad \beta, \varphi \neq 0 .
$$

The condition $\operatorname{Im} N_{J}=\Pi^{2}$ is equivalent to the system

$$
\left\{\begin{array}{l}
\varphi \partial_{\xi_{2}} \alpha=\alpha \partial_{\xi_{1}} \alpha-\frac{1+\alpha^{2}}{\beta} \partial_{\xi_{1}} \beta+\left[a_{1}^{1}\left(1+\alpha^{2}\right)-a_{1}^{2} \alpha \frac{1+\alpha^{2}}{\beta}+a_{2}^{1} \alpha \beta-a_{2}^{2}\left(1+\alpha^{2}\right)\right] \\
\varphi \partial_{\xi_{2}} \beta=\beta \partial_{\xi_{1}} \alpha-\alpha \partial_{\xi_{1}} \beta+\left[a_{1}^{1} \alpha \beta+a_{1}^{2}\left(1-\alpha^{2}\right)+a_{2}^{1} \beta^{2}-a_{2}^{2} \alpha \beta\right]
\end{array}\right.
$$

and the inequality $\left(\partial_{\eta_{1}} \varphi-b_{1} \alpha-b_{2} \beta\right)^{2}+\left(\partial_{\eta_{2}} \varphi-b_{1} \frac{1-\alpha^{2}}{\beta}+b_{2} \alpha\right)^{2}>0$. The system is in the Cauchy-Kovalevskaya form and so possesses a local solution. After this the inequality is arranged to hold.

THEOREM 3. Nijenhuis tensor characteristic distributions in the domain of non-integrability for $J$ have the same singularities as the usual two-dimensional distributions in $\mathbb{R}^{4}$. 
Proof. Let us first define the degeneration locus of a distribution. Introduce the partial order on the growth vectors: $\left(m_{1}, \ldots, m_{s}\right) \leq\left(n_{1}, \ldots, n_{r}\right)$ iff $s \geq r$ and $m_{i} \leq n_{i}$ for $i=1, \ldots, r$. Fix one growth vector $I$. Then the degeneration locus $\Sigma_{I} \subset M$ is the set of points with the growth vector less than or equal to $I$. Proposition 2 (it holds formally as well - on the jets of the structure) and the Thom transversality theorem imply that for a typical $J$ the sets $\Sigma_{I}$ are nice subvarieties, stratifying the manifold $M$. The statement follows.

The generic degenerations of two-plane fields in $\mathbb{R}^{4}$, up to codimension 3 , were classified by Zhitomirskii [Z]. Let us show how generic codimension 2 singularities are realized as a Nijenhuis tensor characteristic distribution.

There are two different types of such singularities, defined by the growth vectors $I_{1}=(2,2,4)$ and $I_{2}=(2,3,3,4)$. All other growth vectors are subordinated to these two and hence the singular set is

$$
\Sigma=\Sigma_{1}^{2} \cup \Sigma_{2}^{2}, \quad \Sigma_{i}^{2}=\Sigma_{I_{i}} .
$$

Generically the loci $\Sigma_{i}^{2}$ are smooth 2-dimensional submanifolds ([Z]), which intersect nontransversally along a curve $\Sigma_{1}^{1}$. There is also a curve $\Sigma_{2}^{1} \subset \Sigma_{2}^{2}$ separating the locus into the elliptic/hyperbolic parts $\Sigma_{2 \pm}^{2}$.

The codimension 2 loci of $\Pi^{2}=\left\langle\xi_{1}, \xi_{2}\right\rangle$ have the following normal forms:

$$
\begin{aligned}
\Sigma_{1}^{2} \backslash \Sigma_{1}^{1}: & \xi_{1}=\partial_{3}, \quad \xi_{2}=\partial_{4}-x_{3} x_{4} \partial_{2}-x_{3}^{2} \partial_{1} ; \\
\Sigma_{2+}^{2}: & \xi_{1}=\partial_{3}, \quad \xi_{2}=\partial_{4}-\left(\frac{1}{3} x_{3}^{3}+x_{3} x_{4}^{2}\right) \partial_{2}-x_{3} \partial_{1} ; \\
\Sigma_{2-}^{2}: & \xi_{1}=\partial_{3}, \quad \xi_{2}=\partial_{4}-x_{3}^{2} x_{4} \partial_{2}-x_{3} \partial_{1} .
\end{aligned}
$$

In each of these cases the choice $\eta_{1}=\partial_{1}, \eta_{2}=\partial_{2}$ and formula (2) will lead to realization $\Pi^{2}=\operatorname{Im} N_{J}$. The cases of higher degenerations are studied similarly.

4. Singularities of $\Pi=\operatorname{Im} N_{J}$ as of a differential system. As differential systems Nijenhuis tensor characteristic distributions have singularities different from those of the usual differential systems in $\mathbb{R}^{4}$ : The rank of a Nijenhuis tensor characteristic distribution is even and so is 2 or 0 .

Proposition 4. For a generic structure $J$ the set where $N_{J}=0$ (the rank of $\Pi$ falls to zero) is a discrete set $\Sigma^{0} \subset M^{4}$. For each point of $\Sigma^{0}$ there is a centered coordinate neighborhood $\left(x_{1}, y_{1}, x_{2}, y_{2}\right)$ around it such that the almost complex structure is given by the formula

$$
J \partial_{x_{i}}=\alpha_{i} \partial_{x_{i}}+\left(1+\beta_{i}\right) \partial_{y_{i}}, \quad J \partial_{y_{i}}=-\frac{1+\alpha_{i}^{2}}{1+\beta_{i}} \partial_{x_{i}}-\alpha_{i} \partial_{y_{i}}, \quad i=1,2,
$$

where the functions $\alpha_{i}, \beta_{i}$ are of the second order of smallness at the origin.

Proof. Singularities of the differential system $\Pi=\operatorname{Im} N_{J}$ are given by the vector equation $N_{J}(\xi, \eta)=0$ for some $J$-independent vector fields $\xi, \eta$, and so are generically isolated points given by the integrability condition $N_{J}=0$.

To get the other claim recall $([\mathrm{K} 1])$ that an almost complex structure can be approximated by a complex structure to the second order of smallness at the integrability points. 
Let $\left(w_{1}, w_{2}\right)$ be the corresponding complex coordinates. By a theorem of Nijenhuis and Woolf $[\mathrm{NW}$ ( see also Proposition 9 below) there are two $J$-holomorphic foliations by disks $C^{1}$-close to the foliations $\left\{w_{i}=\right.$ const. $\}$ at the origin, $i=1,2$. Let $z_{1}$ be a complex coordinate on the disk of the first family passing the origin and $z_{2}$ - on the second. They define the complex coordinate system $\left(z_{1}, z_{2}\right)$ in a neighborhood of the origin with the required properties.

REMARK 1. For $\operatorname{dim} M>4$ the set where $N_{J}=0$ is generically empty.

Let $\alpha_{i}^{\diamond}, \beta_{i}^{\diamond}$ be the quadratic parts of $\alpha_{i}, \beta_{i}$. Using the coordinate system from Proposition 4 we calculate: $\Pi^{2}=\operatorname{Im} N_{J}=\left\langle\xi_{1}, \xi_{2}=J \xi_{1}\right\rangle$, where linearizations of the generators at the origin are

$$
\xi_{1}^{0}=\left(-\frac{\partial \beta_{1}^{\diamond}}{\partial x_{2}}-\frac{\partial \alpha_{1}^{\diamond}}{\partial y_{2}}\right) \partial_{x_{1}}+\left(\frac{\partial \alpha_{1}^{\diamond}}{\partial x_{2}}-\frac{\partial \beta_{1}^{\diamond}}{\partial y_{2}}\right) \partial_{y_{1}}+\left(\frac{\partial \beta_{2}^{\diamond}}{\partial x_{1}}+\frac{\partial \alpha_{2}^{\diamond}}{\partial y_{1}}\right) \partial_{x_{2}}+\left(\frac{\partial \beta_{2}^{\diamond}}{\partial y_{1}}-\frac{\partial \alpha_{2}^{\diamond}}{\partial x_{1}}\right) \partial_{y_{2}}
$$

and $\xi_{2}^{0}=J_{0} \xi_{1}^{0}$ ( $J_{0}$ is the constant coordinate extension of $J$ from the origin).

Thus we see that the linearization of the considered differential system is special, not as for the usual differential systems. If we consider linear vector fields $\xi_{i}^{0}$ as linear operators, we represent the first order approximation of $\Pi$ by a two-dimensional subspace $V^{2} \subset \operatorname{gl}(4)$. The condition $V^{2}=\left\langle X_{1}, X_{2}=J X_{1}\right\rangle$ for some $J^{2}=-\mathbf{1}$ characterizes admissible 2-planes and thus linearizations. The higher order terms in $\xi_{1}, \xi_{2}$ are special as well.

5. Moduli of a PH-curve neighborhood. Let $\mathcal{C}^{2}$ be a pseudoholomorphic (PH-) curve, i.e. a surface with $J$-invariant tangent bundle. At every point $x \in \mathcal{C}$ we have two $J$-invariant planes $T_{x} \mathcal{C}^{2}$ and $\Pi_{x}^{2}$, which generically intersect by zero, except at a finite number of points $\Sigma_{0}^{\prime} \subset \mathcal{C}$. The sets $\Sigma_{1}^{\prime}=\Sigma_{1}^{2} \cap \mathcal{C}$ and $\Sigma_{2}^{\prime}=\Sigma_{2}^{2} \cap \mathcal{C}$ are generically finite as well. The arrangement of all these points

$$
\Sigma^{\prime}=\Sigma_{0}^{\prime} \cup \Sigma_{1}^{\prime} \cup \Sigma_{2}^{\prime} \subset \mathcal{C}
$$

gives a (finite-dimensional) invariant of $\mathcal{C}$.

For points $x \in \mathcal{C} \backslash \Sigma_{1}^{\prime}$ we define field of directions $L^{1}=T \mathcal{C} \cap \Pi^{3}$. The integral curves of this 1-distribution foliate the set $\mathcal{C} \backslash \Sigma_{1}^{\prime}$ and in general $\mathcal{C}$ foliates with only non-degenerate singular points. Denote the number of elliptic points by $e\left(L^{1}\right)$ and the number of hyperbolic points by $h\left(L^{1}\right)$. One can prove:

Proposition 5. Under $C^{1}$-small perturbation of the structure $J$ the foliation $L^{1}$ has minimal number of singularities: $\min \{e(L), h(L)\}=0, \max \{e(L), h(L)\}=|\chi(\mathcal{C})|$. For instance if $\mathcal{C}=T^{2}$ we get a foliation without singularities.

Due to Section 2 the foliation $L^{1}$ is oriented, cooriented and has parallel and transverse measures outside $\Sigma^{\prime}$. Thus there exist canonical vector fields $v_{1}$ along $L^{1}$ and $v_{2}=J v_{1}$ transverse to it. Consequently the curve $\mathcal{C}$ has a lot of dynamical invariants like winding classes of $v_{1}$ and $v_{2}$. Moreover, decomposing

$$
\left[v_{1}, v_{2}\right]=\gamma_{1} v_{1}+\gamma_{2} v_{2}
$$

we obtain two invariant (under pseudoholomorphic isomorphisms) functions $\gamma_{1}, \gamma_{2}$. These together with the germs of the functions $c_{j k}^{i}$ from Section 2 form moduli of the $\mathcal{C}$-neigh- 
borhoods germ. They solve the equivalence problem for PH-embeddings $\mathcal{C}^{2} \rightarrow M^{4}$ (of general position).

EXAMPLE. Let $M=T^{2}(\varphi, \psi) \times \mathbb{R}^{2}(x, y)$ be equipped with the structure

$$
\begin{aligned}
& J \partial_{x}=\partial_{y} ; \quad J \partial_{\varphi}=\frac{2-\rho y^{2}}{2} \partial_{\psi}+\frac{y^{2}}{2} \partial_{\varphi}+x \partial_{x} ; \\
& J \partial_{y}=-\partial_{x} ; \quad J \partial_{\psi}=\frac{4+y^{4}}{2 \rho y^{2}-4} \partial_{\varphi}-\frac{y^{2}}{2} \partial_{\psi}+\frac{x y^{2}}{\rho y^{2}-2} \partial_{x}+\frac{2 x}{\rho y^{2}-2} \partial_{y} .
\end{aligned}
$$

Then $\mathcal{C}=\{x=y=0\}$ is a PH-torus and the winding number of $v_{1}$ is $\rho$. Similarly one shows the other considered invariants are non-trivial.

6. Hyperbolicity of a PH-curve neighborhood. In this section we consider the case of PH-tori $\mathcal{C}=T^{2}$. We assume for simplicity that the normal bundle is topologically trivial, though in general case the result is the same.

Recall that the Kobayashi pseudometric $d_{M}$ measures the distance between points via pseudoholomorphic disks ([Ko, KO ]). An almost complex manifold is called Kobayashi hyperbolic if $d_{M}$ is a metric. Let $\|\cdot\|$ be a norm on $T M$.

Proposition 6. Let $\mathcal{O}$ be a small neighborhood of a pseudoholomorphic torus $T^{2} \subset\left(M^{4}, J\right)$. Then the domain $\mathcal{O} \backslash T^{2}$ is not Kobayashi-hyperbolic.

Moreover, for some constant $C>0$ and any $R>0$ there exists a smooth family of PH-disks $f_{\alpha}^{R}: D_{R} \rightarrow \mathcal{O}$, with uniformly bounded norms $\left\|\left(f_{\alpha}^{R}\right)_{*}(z)\right\| \leq C$ satisfying $\left\|\left(f_{\alpha}^{R}\right)_{*}(0)\right\|=1$, that fills some smaller neighborhood $\mathcal{O}^{\prime} \subset \mathcal{O}$ of $T^{2}$ :

$$
\mathcal{O}^{\prime} \subset \bigcup_{\alpha} f_{\alpha}^{R}\left(D_{R}\right)
$$

Proof. Let us take the universal covering $\hat{\mathcal{O}} \simeq \mathbb{C} \times D^{2}$ of $\mathcal{O}$. The torus is covered by the entire line $\mathbb{C} \rightarrow T^{2}$. Changing the structure $J$ at infinity in $\hat{\mathcal{O}}$ and near the boundary to the integrable one we glue the manifold to the product $S^{2} \times S^{2}$ with the line $\mathbb{C}$ being glued to the first factor $S_{1}^{2}$. Then the introduction of the taming symplectic product structure $\omega=\omega_{1} \oplus \omega_{2}$ yields a foliation of $S_{1}^{2} \times S_{2}^{2}$ by PH-spheres $S^{2}$ in the homology class of the first factor if we additionally demand that the homology class $\left[S_{1}^{2}\right]$ of the first sphere-factor is symplectically indecomposable (for example, if $\omega_{1}\left(S_{1}^{2}\right)=k \omega_{2}\left(S_{2}^{2}\right)$, $k \in \mathbb{N}$ ). Here we use the fact that the dimension is 4 : due to positivity of intersections [M1] we actually have a foliation ([M2]).

This foliation of $S^{2} \times S^{2}$ gives a family of big $\mathrm{PH}$-disks on $\hat{\mathcal{O}}$ parametrized by the radius $R$ of disk in $\mathbb{C}$ out of which the almost complex structure is changed. The estimates follow from the Brody reparametrization lemma as in [KO]. Pulling-back we get the required family.

We now consider filling by pseudoholomorphic cylinders $\mathcal{C}_{R}=[-R ; R] \times S^{1} \subset \mathbb{C} \backslash\{0\}$, which is topologically different from the disk-filling (Fig. 1).

Proposition 7. In the statement of Proposition 6 we can change disks $D_{R}$ to the cylinders $\mathcal{C}_{R}$ and get for every $R>0$ a filling family of PH-cylinders $f_{\alpha}^{R}: \mathcal{C}_{R} \rightarrow \mathcal{O}$ with 
uniformly bounded norms and normalization $\left\|\left(f_{\alpha}^{R}\right)_{*}(0)\right\|=1$ :

$$
\mathcal{O}^{\prime} \subset \bigcup_{\alpha} f_{\alpha}^{R}\left(\mathcal{C}_{R}\right)
$$



Figure 1. Filling by PH-cylinders

Proof. Actually take a covering of the neighborhood $\mathcal{O}$ which corresponds to one cycle of the torus. The torus is covered by the entire cylinder $\mathcal{C}_{\infty} \rightarrow T^{2}$. We can change the almost complex structure $J$ at infinity so that it makes possible to "pinch" each end of the cylinder. This means we perturb the structure $J$ so that it is standard integrable outside some $\mathcal{C}_{R_{2}} \subset \mathcal{C}_{\infty}$ and the support is also a big cylinder $\mathcal{C}_{R_{1}}$. Then we glue the ends to the disks. This operation gives us a sphere $S^{2}$ instead of the cylinder $\mathcal{C}_{\infty}=\mathbb{R} \times S^{1}$. We can also assume that neighborhoods of two cylinder ends are pinched (Fig. 2).

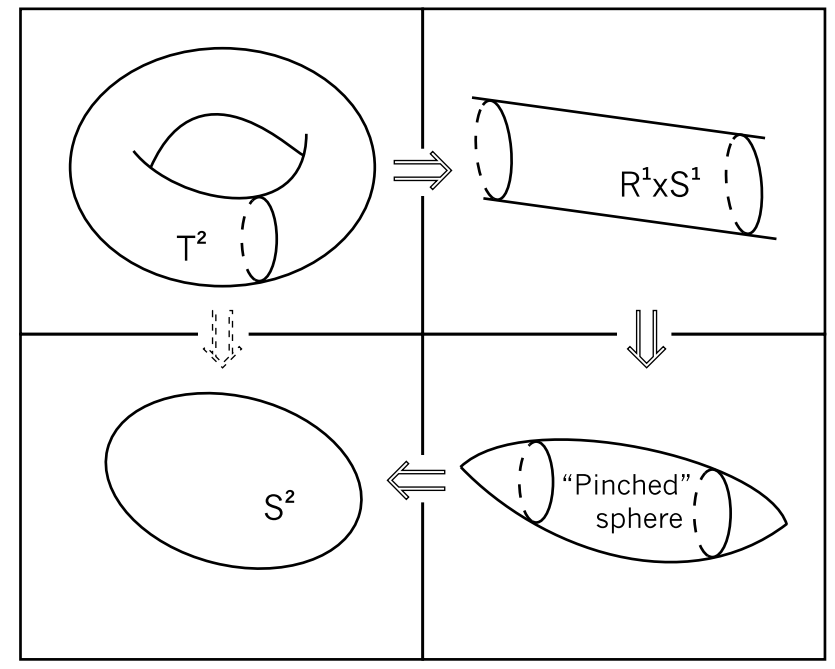

Figure 2. Cutting and gluing

Thus we have a neighborhood $U$ of the sphere $S_{0}^{2}$. It is foliated by PH-spheres close to $S_{0}^{2}$. Actually, we can change the structure $J$ near the boundary of this neighborhood, glue and get the manifold product $\hat{M}=S^{2} \times S^{2}$. As before it is foliated by PH-spheres. Thus $U$ is foliated by $\mathrm{PH}$-spheres and in the preimage they give a $\mathrm{PH}$-foliation by cylinders. 
REMARK 2. Neighborhoods of PH-spheres $\mathcal{C}=S^{2}$ are also non-hyperbolic and if the normal bundle is topologically trivial can be foliated by close PH-spheres. Small neighborhood of PH-curves of higher genus $\mathcal{C}=S_{g}^{2}, g>1$, are Kobayashi hyperbolic.

Moreover the above non-hyperbolicity for the cases $g=0,1$ can be strengthened as follows: Even the punctured neighborhoods $\mathcal{O} \backslash \mathcal{C}$ are non-hyperbolic.

7. Arnold's question. In [A2] (1993-25) Arnold asks about almost complex version for his Floquet-type theory of elliptic curves neighborhoods ([A1]) in the spirit of the Moser's KAM-type theorem ([Mo]). Namely he asks if a germ of neighborhood $\mathcal{O}$ of a PH-torus $\mathcal{C}=T^{2} \subset\left(M^{4}, J\right)$ is determined by its normal bundle $N_{\mathcal{C}} M$.

The following result is a direct consequence of the definition:

Proposition 8. If $F: M^{4} \rightarrow \mathcal{C}^{2}$ is a (local) PH-surjection and the structure $J$ is non-integrable, then the Nijenhuis tensor characteristic distribution $\Pi^{2}$ is integrable and is tangent to the fibers of $F$.

Thus there is a functional obstruction to the equivalence of the $\mathcal{C}$-germ in $M^{4}$ and of the $\mathcal{C}$-germ in the normal bundle (we do not discuss here the normal bundle: If $\operatorname{dim} M=4$, the almost complex structure on $N_{\mathcal{C}} M$ can be obtained via linearization along a family of transversal PH-disks; for the general case see [K3]). Integrability and transversality of $\Pi^{2}$ to the torus $\mathcal{C}$ is a necessary, but by no means sufficient condition for the existence of an equivalence: There are other functional moduli.

In search of a proper generalization of Arnold's result we notice that a neighborhood of an elliptic curve in a complex surface is foliated by half-infinite cylinders: They are given as $|z|=$ const. in the representation of the neighborhood as $\mathbb{C}^{2}(z, w) /(z, w) \sim$ $(z+2 \pi, w) \sim(z+\nu, \lambda w)$, where $\nu \in \mathbb{C} \backslash \mathbb{R}$ and $\lambda \in \mathbb{C} \backslash\{0\}$ (see [A1] for the representation). The hypothesis is then that for a non-integrable perturbation $J$ of the complex structure $J_{0}$ most of the cylinders persist (as in Moser's theory).

Let us sketch how to prove existence of one such a half-cylinder. In Proposition 7 we have constructed a pre-compact family of finite cylinders $f_{\alpha}^{R}$ for different $R$. If it winds up to the curve $\mathcal{C}$ (as in the holomorphic normal form with $|\lambda| \neq 1$ ), then one can extract a subsequence $f_{\alpha_{k}}^{R_{k}}$ with $R_{k} \rightarrow \infty$ converging to a pseudoholomorphic curve due to the standard technique $([\mathrm{G}, \mathrm{MS}])$. This is the required half-cylinder.

There are no tools however to complete this construction to a $\mathrm{PH}$-foliation (also a filling is problematic - a remark of V. Bangert). Note though that even if we construct a foliation, it is not necessarily so nice as its holomorphic original. To explain this let us notice the following fact, which is a corollary of a theorem by Nijenhuis and Woolf [NW]:

Proposition 9. A small neighborhood $\mathcal{O}$ of a PH-curve $\mathcal{C} \subset M^{4}$ can be foliated by transversal PH-disks $D^{2}$.

Now consider a neighborhood of a $\mathrm{PH}$-curve $\mathcal{C}$ with topologically trivial normal bundle and suppose we have a foliating family $f_{\alpha}: \mathcal{B} \rightarrow \mathcal{O}$ with unbounded or compact leaves in it. Let $D_{\varphi}, \varphi \in \mathcal{C}$, be the family of normal disks from Proposition 9 . Then every path $\gamma(t)$ on $\mathcal{C}$ with $\gamma(0)=\varphi_{0}, \gamma(1)=\varphi_{1}$ gives a mapping $\Phi_{\gamma}: D_{\varphi_{0}} \rightarrow D_{\varphi_{1}}$ of shift along the leaves of $f_{\alpha}$. For a loop $\gamma$ we have an automorphism of $D_{\varphi}$. Since $f_{\alpha}$ is a foliation there 
is no local holonomy: $\Phi_{\gamma}=$ id for contractible loops $\gamma$. Thus we can consider the map $\pi_{1}(\mathcal{C}) \rightarrow \operatorname{Aut}\left(D_{\varphi}\right)$.

Definition 2. We call $\Phi_{\gamma} \in \operatorname{Aut}\left(D_{\varphi}\right)$ the monodromy map along $\gamma \in \pi_{1}(\mathcal{C})$ and $\Phi_{\gamma}: D_{\varphi_{0}} \rightarrow D_{\varphi_{1}}$ the transport map.

For example there is no monodromy for the sphere $\mathcal{C}=S^{2}$ and each choice of local coordinates in a normal disk $D_{\varphi_{0}}$ gives coordinates for the others $D_{\varphi}$.

Let now $\mathcal{C}=T^{2}(2 \pi, \nu)$ and we have a foliating family $f_{\alpha}$ of half-infinite cylinders. Since every leaf $\mathcal{B}$ is a cylinder, there is no monodromy along one generating cycle. Normalize it to be the cycle $\varphi \mapsto \varphi+2 \pi$. Denote by $\Phi_{\nu}$ the monodromy along the other cycle $\varphi \mapsto \varphi+\nu$.

Unlike the complex case, the almost complex monodromy can be non-holomorphic mapping of the fibers: It is possible to construct examples of $\mathrm{PH}$-foliations with any prescribed monodromy $\Phi_{\nu}$.

Moreover even if the monodromy is complex, the transport maps $\Phi_{\gamma}:\left(D_{\varphi_{0}}, J\right) \rightarrow$ $\left(D_{\varphi_{1}}, J\right)$ can be non-complex. In fact there are functional obstructions for the transports to be complex:

Theorem 10. Let $\mathcal{C}$ be a PH-curve in a 4-dimensional manifold $(M, J)$ and let $f_{\alpha}: \mathcal{B} \rightarrow \mathcal{O}$ be a local PH-foliating family in some neighborhood $\mathcal{O}$ of $\mathcal{C}$. Then if all transport maps $\Phi_{\gamma}$ are holomorphic, then the Nijenhuis tensor characteristic distribution $\Pi^{2}$ is integrable and is tangent to the leaves of $f_{\alpha}$.

Proof. Actually this is because the foliation provides a local bundle $\pi: \mathcal{O} \rightarrow D_{\varphi}$ and so Proposition 8 applies.

Again the integrability is not a sufficient condition: There are other moduli.

So we see that the existence of foliating $\mathrm{PH}$-family with complex transports (as in the original holomorphic case) is generically obstructed, and the obstructions are of the same nature as for the existence of equivalence between a germ of a neighborhood of a PHcurve $\mathcal{C}$ and its normal bundle (though in the first case the Nijenhuis tensor characteristic distribution is tangent to the curve $\mathcal{C}$, while in the second one it is transversal).

\section{References}

[A1] V. I. Arnol'd, Supplementary chapters to the theory of ordinary differential equations, Nauka, Moscow, 1978 (Russian); English transl.: Geometrical Methods in the Theory of Ordinary Differential Equations, Springer, New York, 1988.

[A2] Arnol'd's Problems, FAZIS, Moscow, 2000 (Russian); English transl. to appear.

[E] F. Engel, Zur Invariantentheorie der Systeme von Pfaff'schen Gleichungen, Berichte Ges. Leipzig, Math-Phys. Classe XLI (1889), 157-176.

[G] M. Gromov, Pseudo-holomorphic curves in symplectic manifolds, Invent. Math. 82 (1985), 307-347.

[HH] F. Hirzebruch, H. Hopf, Felder von Flächenelementen in 4-dimensionalen Mannigfaltigkeiten, Math. Ann. 136 (1958), 156-172. 
[Ko] S. Kobayashi, Hyperbolic Manifolds and Holomorphic Mappings, Marcel Dekker, New York, 1970.

[KO] B. Kruglikov, M. Overholt, Pseudoholomorphic mappings and Kobayashi hyperbolicity, Differential Geom. Appl. 11 (1999), 265-277.

[K1] B. S. Kruglikov, Nijenhuis tensors and obstructions for pseudoholomorphic mapping constructions, Mat. Zametki 63 (1998), 541-561 (Russian); English transl.: Math. Notes 63 (1998), 476-493.

[K2] B. S. Kruglikov, Some classificational problems in four-dimensional geometry: distributions, almost complex structures and Monge-Ampère equations, Mat. Sb. 189:11 (1998), 61-74 (Russian); English transl.: Sb. Math. 189 (1998), 1643-1656.

[K3] B. S. Kruglikov, Nijenhuis tensors in pseudoholomorphic curves neighborhoods, University of Troms $\varnothing$, preprint 00-40 (2000); e-print: http://arXiv.org/abs/math.DG/0105130

[M1] D. McDuff, The local behavior of holomorphic curves in almost complex 4-manifolds, J. Differential Geom. 34 (1991), 143-164.

[M2] D. McDuff, Blow ups and symplectic embeddings in dimension 4, Topology 30 (1991), 409-421.

[MS] D. McDuff, D. Salamon, J-holomorphic Curves and Quantum Cohomology, Univ. Lecture Ser. 6, Amer. Math. Soc., Providence, 1994.

[Mo] J. Moser, On the persistence of pseudo-holomorphic curves on an almost complex torus, Invent. Math. 119 (1995), 401-442.

[NW] A. Nijenhuis, W. Woolf, Some integration problems in almost-complex and complex manifolds, Ann. of Math. (2) 77 (1963), 424-489.

[S] S. Sternberg, Lectures on Differential Geometry, Prentice-Hall, Englewood Cliffs, 1964.

[Z] M. Ya. Zhitomirskii, Normal forms of germs of two-dimensional distributions on $R^{4}$, Funktsional. Anal. i Prilozhen. 24:2 (1990), 81-82 (Russian); English transl.: Funct. Anal. Appl. 24 (1990), 150-152. 